Zwischen Taktstock und Hörsaal 
QUELLEN UND STUDIEN ZUR MUSIK IN BADEN-WÜRTTEMBERG

herausgegeben von der

GESELLSCHAFT FÜR MUSIKGESCHICHTE

IN BADEN-WÜRTTEMBERG e.V.

Band 3 
Gabriela Rothmund- Gaul

\section{Zwischen Taktstock und Hörsaal}

Das Amt des Universitätsmusikdirektors in Tübingen 1817 - 1952

Verlag J. B. Metzler Stuttgart - Weimar 


\section{GESELLSCHAFT FÜR MUSIKGESCHICHTE IN BADEN-WÜRTTEMBERG e.V.}

Schulberg 2, 72070 Tübingen

Präsident: Prof. Dr. Rolf Keller

Vizepräsident: Heinz Silberhorn

Vorsitzender des Wissenschaftlichen Beirats: Prof. Dr. Manfred Hermann Schmid

Die Deutsche Bibliothek - CIP-Einheitsaufnahme

\section{Rothmund-Gaul, Gabriela:}

Zwischen Taktstock und Hörsaal :

das Amt des Universitätsmusikdirektors in Tübingen 1817 - 1952 /

Gabriela Rothmund-Gaul. - Stuttgart ; Weimar, 1998

(Quellen und Studien zur Musik in Baden-Württemberg ; Bd. 3)

ISBN 978-3-476-01599-0

ISBN 978-3-476-03741-1 (eBook)

DOI 10.1007/978-3-476-03741-1

Dieses Werk einschließlich aller seiner Teile ist urheberrechtlich geschützt. Jede Verwertung außerhalb der engen Grenzen des Urheberrechtsgesetzes ist ohne Zustimmung des Verlages unzulässig und strafbar. Das gilt insbesondere für Vervielfältigungen, Übersetzungen, Mikroverfilmungen und die Einspeicherung und Verarbeitung in elektronischen Systemen.

(C) 1998 Springer-Verlag GmbH Deutschland Ursprünglich erschienen bei J. B. Metzlersche Verlagsbuchhandlung und Carl Ernst Poeschel Verlag GmbH in Stuttgart 1998 
Inhaltsverzeichnis

Vorwort $\mathrm{XI}$

Abkürzungen XII

Ämter, Behörden und Institutionen XIV

Einleitung 1

I. „...Die Aufstellung eines Musiklehrers an der Universität betreffend «

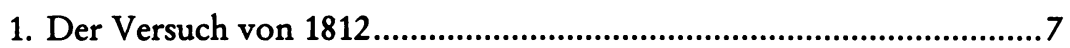

1.1. Wangenheims Musiklehrer-Idee .................................................... 8

1.2. Die Überlegungen auf ministerieller Ebene .................................. 11

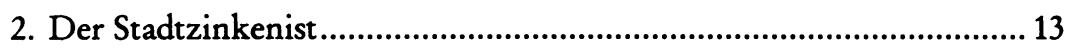

2.1. Das Verhältnis zwischen Stadtzinkenist und

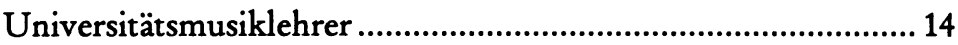

2.1.1. Die Paragraphen von 1852 ...................................................... 14

2.1.2. Das Statut von 1869................................................................. 14

2.1.3. Der Vertrag von 1872 ................................................................. 16

2.1.4. Reduktion der Verpflichtungen ................................................. 17

2.1.5. Ende des Vertrags mit der Universität ....................................... 19

3. Die Klage über die Kirchenmusik im Jahr 1813 .................................. 20

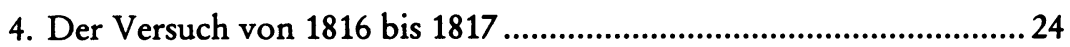

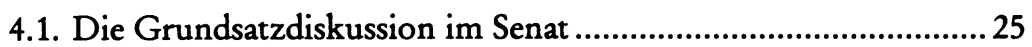

4.2. Kultminister Wangenheims Anliegen ...........................................28

4.3. Jonathan Friedrich Bahnmaier ................................................... 30

4.3.1. Bahnmaiers Position im Senat .................................................. 31

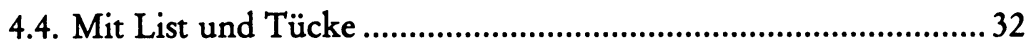

II. Universitätsmusikdirektor Friedrich Silcher.............................. 35

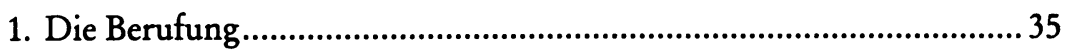

1.1. Bahnmaiers Einfluß im Senat ......................................................36

1.2. Bahnmaiers Musiklehrer-Idee ......................................................36

1.3. Friedrich Silcher ........................................................................... 39

1.4. Die Wende im Senat ..................................................................... 41

1.5. Die Ernennung Silchers................................................................. 43 
2. Friedrich Silcher im Amt ....................................................................... 43

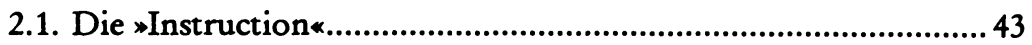

2.1.1. Die Stellung des Universitätsmusikdirektors ............................ 45

2.1.2. Die Pflichten am Seminar .......................................................... 46

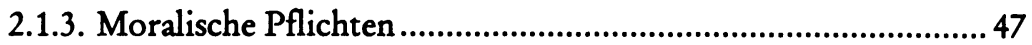

2.2. Die Zusatzinstruktion für das Seminar...................................... 47

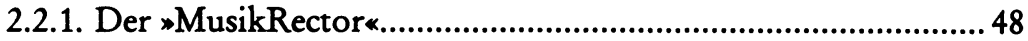

2.2.2. Der Universitätsmusikdirektor.................................................. 49

2.3. Verpflichtung an den niederen Seminaren.................................50

2.4. Silchers Amt am Wilhelmsstift .................................................51

2.4.1. Silchers Unterrichtsentwurf..................................................... 53

2.4.2. Hindernisse................................................................................. 53

3. Das Amt zum Zeitpunkt von Silchers Pensionierung.........................58

3.1. Die Pflichten am Seminar........................................................59

3.2. Die Leitung der Kirchenmusik ......................................................60

3.3. Die Pflichten am Predigerinstitut ................................................. 61

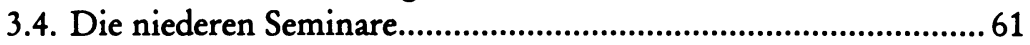

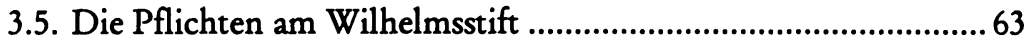

3.6. Die Pflichten gegenüber der Universität........................................ 64

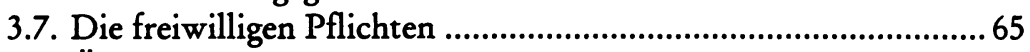

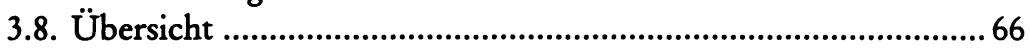

3.9. Besoldung und Anerkennung .......................................................67

3.9.1. Verleihung der Ehrendoktorwürde ............................................6 68

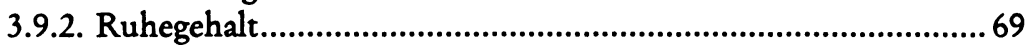

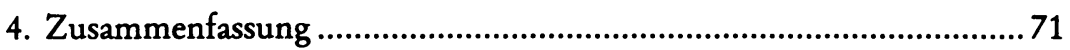

II. Universitätsmusikdirektor Otto Scherzer..................................73

1. Die Berufung.....................................................................................73

1.1. Theologieprofessor Christian Palmer ..........................................73

1.2. Senatsreferent Christian Palmer .................................................. 75

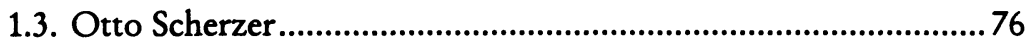

1.4. Die Verhandlung im Senat ........................................................... 82

1.4.1. Die Definition der Stelle............................................................. 82

1.4.2. Die Pflichten am Seminar, am Wilhelmsstift und

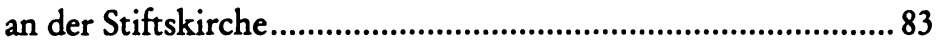

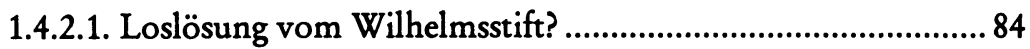

1.4.2.2. Loslösung vom Evangelischen Seminar?..................................85

1.4.2.3. Noch Kirchenmusik? ............................................................... 87

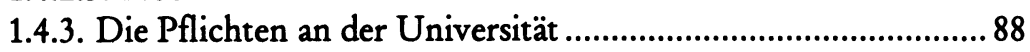

1.4.4. Die Personaldiskussion ............................................................ 90

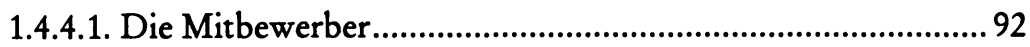

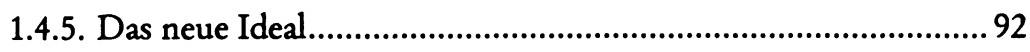

1.4.6. Pädagoge ja oder nein? .............................................................. 93 


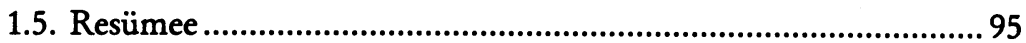

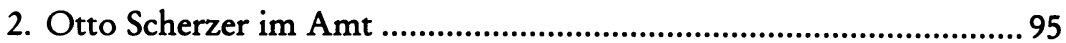

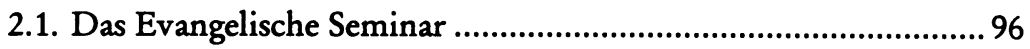

2.1.1. Der Gesangsunterricht............................................................. 98

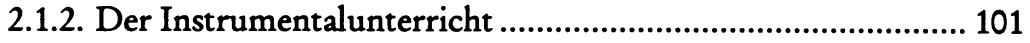

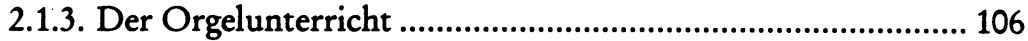

2.2. Die Visitation der niederen Seminare ....................................... 105

2.3. Kirchenmusik an der Stiftskirche .......................................... 106

2.4. Neue Pflichten am Wilhelmsstift............................................ 107

2.5. Die Pflichten an der Universität ............................................... 111

2.5.1. Der Orchesterverein ........................................................... 112

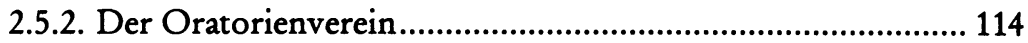

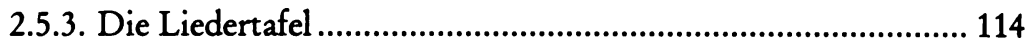

3. Zum Verständnis des Amtes............................................................ 116

3.1. Gehalt und Pension .................................................................. 117

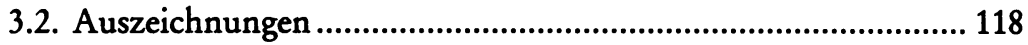

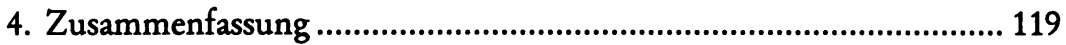

IV. Universitätsmusikdirektor Emil Kauffmann.......................... 121

1. Berufung ........................................................................................... 121

1.1. Emil Kauffmann.................................................................... 122

1.2. Die Verhandlungen mit Emil Kauffmann................................... 123

1.3. Die Definition des Amtes...................................................... 128

1.3.1. Die Pflichten an der Universität ........................................... 128

1.3.2. Die Pflichten an den theologischen Bildungsanstalten ........... 129

1.3.3. Musik an der Stiftskirche? .................................................... 130

1.4. Die Personalfrage ....................................................................... 131

1.4.1. Wunschkandidat Kauffmann ............................................... 132

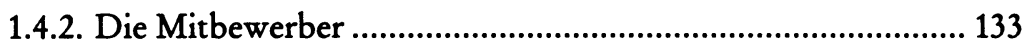

2. Emil Kauffmann im Amt ............................................................ 136

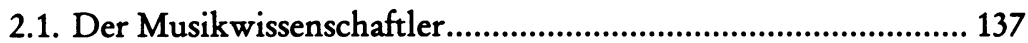

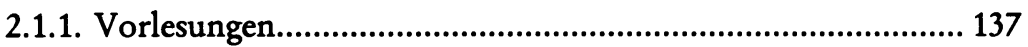

2.1.2. Kauffmanns Promotion ...................................................... 140

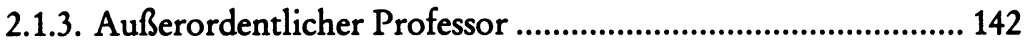

2.2. Der Musiker ............................................................................... 145

2.2.1. Das Evangelische Seminar..................................................... 145

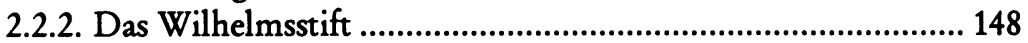

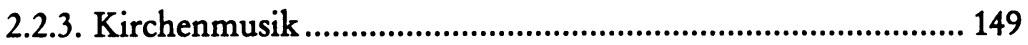

2.2.4. Universitätsfeierlichkeiten ................................................... 150

2.2.5. Die Akademische Liedertafel ................................................ 151

2.2.6. Der Akademische Musikverein .............................................. 152

3. Zusammenfassung .............................................................................. 154 
V. Universitätsmusikdirektor Fritz Volbach ................................ 157

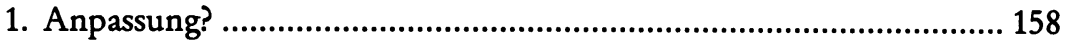

1.1. Musik und Musikwissenschaft an deutschen

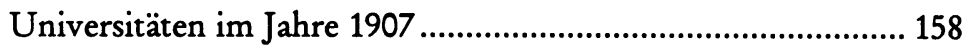

2. Stellenausschreibung und Berufung .............................................. 161

2.1. Die Wünsche der Philosophischen Fakultät ............................. 162

2.2. Das »Schwarze Heft«................................................................. 163

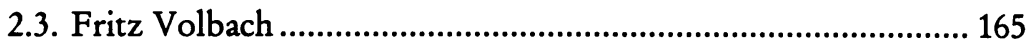

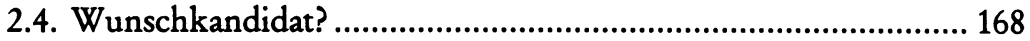

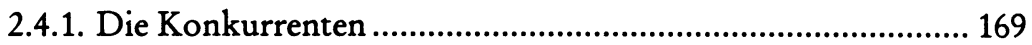

2.4.2. Stimmungsmache gegen Volbach.......................................... 171

2.5. Senatssitzung ............................................................................ 173

2.5.1. Die Aufgaben des Universitätsmusikdirektors........................ 174

2.5.1.1. Die Pflichten am Evangelischen Seminar ............................. 175

2.5.1.2. Die Pflichten am Wilhelmsstift ............................................ 177

2.5.1.3. Die Pflichten an der Universität........................................ 178

2.5.1.4. Die Dirigententätigkeit ................................................... 178

2.5.1.5. Musikwissenschaft ............................................................. 179

2.5.1.6. Private Lehrtätigkeit .......................................................... 180

2.5.2. Die Personaldiskussion ........................................................... 181

2.6. Entscheidung im Ministerium ................................................. 182

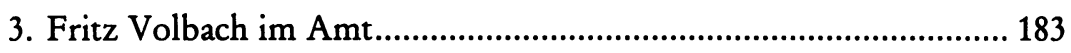

3.1. Das Evangelische Seminar ....................................................... 186

3.1.1. Ein Fall für das Konsistorium? ........................................... 187

3.1.2. Neue Ordnung ohne «Neuordnung «..................................... 188

3.1.3. Erste Schritte zur Reform des Musikunterrichts.................... 189

3.2. Das Wilhelmsstift .................................................................. 192

3.3. Volbachs Angliederung an die Philosophischen Fakultät .......... 193

3.3.1. Streichung als »Lehrer der Künste* im Vorlesungsverzeichnis?....................................................... 196

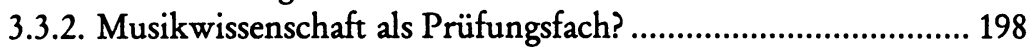

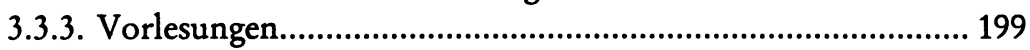

3.4. Der akademische Musikverein.................................................... 200

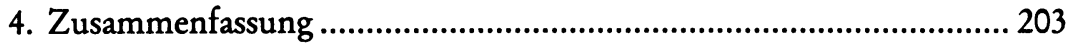

VI. Universitätsmusikdirektor Karl Hasse ................................... 205

1. Das Ende einer Tradition am Evangelischen Seminar...................... 206

1.1. Die Pläne des Konsistoriums .................................................. 207

1.2. Die Pläne der Universität ......................................................... 208

1.3. Konsistorium kontra Universität ............................................. 209

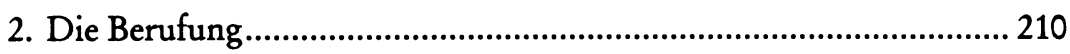




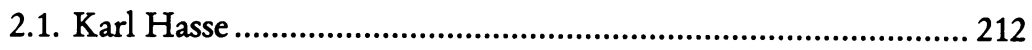

2.2. Die Definition der Stelle............................................................ 214

2.3. Die Personalfrage ................................................................... 217

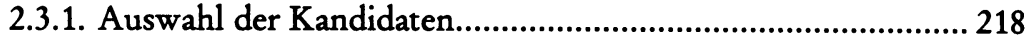

2.3.2. Keller kontra Hasse, Schmid kontra Haller ........................... 222

2.4. Die Entscheidung .................................................................. 225

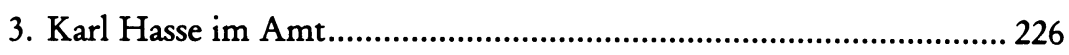

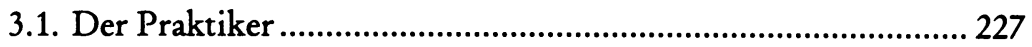

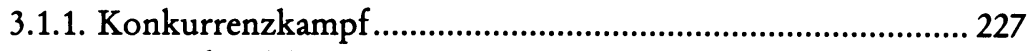

3.1.2. Hasse als Dirigent ................................................................. 231

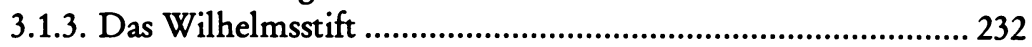

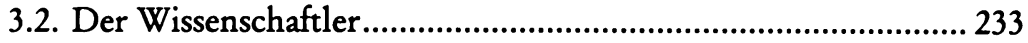

3.2.1. Die Erweiterung des Lehrauftrags.......................................... 233

3.2.2. Musikwissenschaft als Prüfungsfach..................................... 236

3.2.3. Hasses Promotion............................................................. 237

3.2.4. Erweiterung der Räumlichkeiten und Erwerb einer Orgel .... 238

3.2.5. Das Musikinstitut mit musikwissenschaftlichem Seminar ..... 240

3.2.6. Ausbau des Instituts und der eigenen Position...................... 241

3.2.7. Die Veröffentlichungen des Musikinstituts.............................. 243

3.3. Die Bedeutung des Amtes.......................................................... 245

4. Karl Hasse und der Nationalsozialismus ......................................... 248

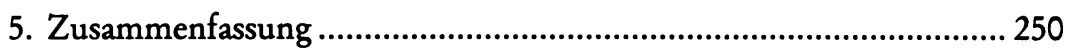

VII. Universitätsmusikdirektor Ernst Fritz Schmid ..................... 253

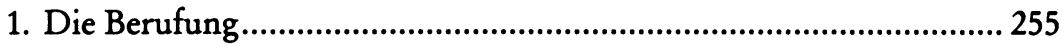

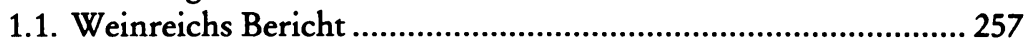

1.1.1. Ernst Fritz Schmid ................................................................ 258

1.1.2. Profil der Stelle ..................................................................... 261

1.1.3. Profil »des künftigen Landschaftsleiters ................................. 264

1.1.4. Kontra aus den eigenen Reihen.............................................. 265

2. Musikwissenschaft - ein Politikum?................................................ 267

3. Ernst Fritz Schmid im Amt.......................................................... 270

3.1. Musizieren unter dem Universitätsmusikdirektor .................... 273

3.2. Das wissenschaftliche Arbeiten ............................................... 278

3.3. Das Schwäbische Landesmusikarchiv ........................................ 280

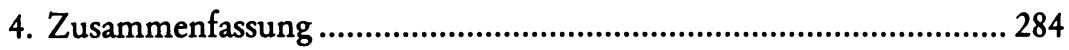

VIII. Universitätsmusikdirektor Carl Leonhardt........................ 287

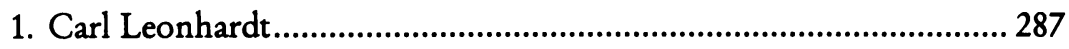

1.1. Leonhardts Abschied von der Stuttgarter Oper......................... 291 
2. Der stellvertretende Universitätsmusikdirektor ............................. 294

2.1. Wünsche der Fakultät .............................................................. 295

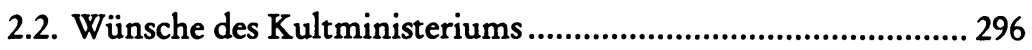

3. Die Suche nach dem neuen Universitätsmusikdirektor .................... 297

3.1. Kampf um Leonhardt.............................................................. 299

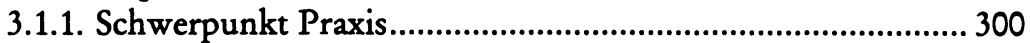

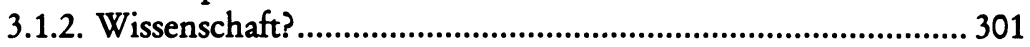

3.2. Druck vom Reichserziehungsministerium................................ 302

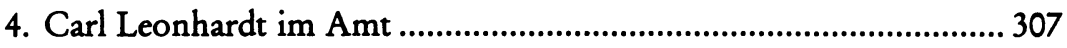

4.1. Der wissenschaftliche Betrieb ................................................. 308

4.2. Der Musiker Leonhardt........................................................ 311

4.2.1. Vom Akademischen Musikverein zum Collegium musicum der Universität ........................................ 313

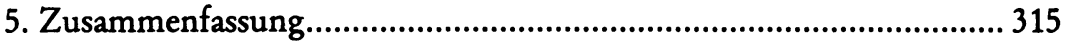

IX. Die Errichtung des planmäßigen Ordinariats........................... 317

1. Vorentscheidung durch die Philosophische Fakultät ......................... 318

2. Das Aus für den Universitätsmusikdirektor .................................... 319

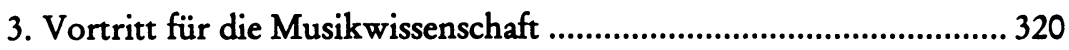

4. Über den persönlichen Ordinarius zum planmäßigen Ordinariat.... 320

»Musikalische Zeitfragen« - Zusammenfassung

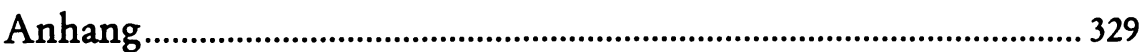

Anbringen des Kurators Wangenheim von 1812................................ 331

Anbringen des Kultministers Wangenheim von 1817 (Original)............ 333

Anbringen des Kultministers Wangenheim von 1817 .............................. 340

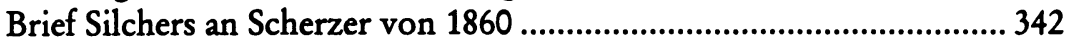

Statut betreffend die Stadtmusikusstelle in Tübingen von 1869............ 347

Artikel aus der Schwäbischen Kronik von 1877 ...................................... 354

Hörer und Doktorandenstatistik des Musikinstituts von 1938 .............. 355

Bewerber auf die Stelle des Universitätsmusikdirektors......................... 356

Übersicht über Vorlesungen, Seminare und Übungen........................... 361

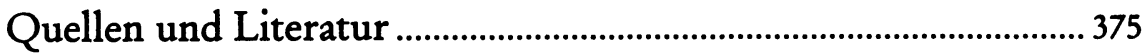

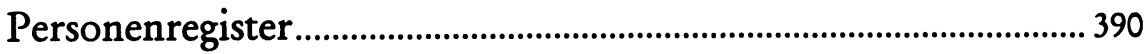




\section{Vorwort}

Die Anregung zur Beschäftigung mit dem Amt des Universitätsmusikdirektors in Tübingen erhielt ich im Sommer 1992 von meinem akademischen Lehrer Prof. Dr. Ulrich Siegele. Zu diesem Zeitpunkt hatten er eine Publikation zur Geschichte der Orgeln im Musikwissenschaftlichen Institut im Bebenhäuser Pfleghof und ich eine Festschrift zu diesem Gebäude fertiggestellt ${ }^{1}$. Mich mit diesem Gebäude, in dem sich der erste Akademische Musiksaal Tübingens befand, mit der Universität, ihrer Geschichte und ganz speziell derjenigen des akademischen Musizierens und seinen institutionellen Voraussetzungen befassen zu können, entsprach meinem Wunsch, den vielen, bei der Entstehung der Festschrift aufgeworfenen Fragen nachzuspüren.

Dazu bedurfte es unter anderem der Recherche in über einem Dutzend Archiven und Bibliotheken. Überall traf ich auf Interesse und fand stets Unterstützung, für die ich mich bei allen Mitarbeiterinnen und Mitarbeitern der im Anhang genannten Archive ausdrücklich bedanke. Es ist mir ein großes Anliegen, ganz besonders den Mitarbeiterinnen und Mitarbeitern des Universitätsarchivs Tübingen meinen Dank auszusprechen, allen voran dem damaligen Direktor des Archivs, Herrn Prof. Dr. Volker Schäfer, Herrn Oberarchivrat Dr. Johannes Michael Wischnath und Frau Irmela Bauer, deren Kenntnis und Hilfsbereitschaft ich schätzen lernen durfte. Ebenso wertvoll war mir die Hilfe der Mitarbeiterinnen und Mitarbeiter des Stadtarchivs Tübingen, im besonderen der stellvertretenden Leiterin Frau Antje Zacharias.

Die angenehmste Pflicht ist es, meinem Doktorvater, Herrn Prof. Dr. Ulrich Siegele, für seine Selbstverständlichkeit zu danken, mit der er von Anfang an mein Unterfangen begleitete, vor allem auch in der Hinsicht, daß er der Überzeugung ist, daß die Bearbeitung der gewählten Thematik gerade nicht allein die Aufgabe der Historiker sein kann, sondern auch bei den $\mathrm{Mu}$ sikwissenschaftlern in den richtigen Händen liegt.

Nicht versäumen möchte ich, auch all denjenigen meine Dankbarkeit auszudrücken, die mir durch Gespräche und das Lesen des Manuskripts wertvolle Hinweise und Anregungen gaben. Dasselbe gilt für die unermüdlichen Helfer, die mir bei der Vorbereitung zum Druck mit Rat und Tat zur Seite standen. Die Zahl der möglichen Fehler wurde dadurch sicher verringert.

Zuletzt danke ich der Gesellschaft für Musikgeschichte in Baden-Württemberg e.V., einerseits für die Aufnahme meiner im Sommer 1996 von der Fakultät für Kulturwissenschaft der Universität Tübingen angenommenen Dissertation in ihre Veröffentlichungsreihe und andrerseits für die finanzielle Unterstützung. Zur Deckung der Druckkosten erfuhr ich außerdem dankenswerterweise großzügige Förderung seitens der Vereinigung der Freunde der Universität Tübingen e.V. (Universitätsbund).

Stuttgart, im Februar $1998 \quad$ Gabriela Rothmund-Gaul

1 Zu den genauen Titeln s. in *Quellen und Literatur» im Anhang. 


\section{Abkürzungen}

$\mathrm{aaO}$

ao.

$\mathrm{ADB}$

AEvST

Akad. RA

AWT

Bd., Bde.

bearb.

Bü

bzw.

DAR

Diss.

DLAM

ebd.

Erl.

erw.

f.

Fak.

ff.

fl.

fol.

geb.

GS

Hf.

Hrsg.

hrsg.

HStASt

Jg.

Jh.

KK

KM

Kons.

Konsist.

KRAT

KS

LKA

LMA

MA

masch.

MGG

NDB am angegebenen Ort

außerordentlicher

Allgemeine Deutsche Biographie

Archiv des Evangelischen Stifts Tübingen

Akademisches Rektoramt

Archiv des Wilhelmsstifts Tübingen

Band, Bände

bearbeitet

Büschel

beziehungsweise

Diözesanarchiv Rottenburg

Dissertation

Deutsches Literaturarchiv Marbach - Schiller

Nationalmuseum

ebenda

Erlaß

erweitert

folgende

Fakultät

die beiden folgenden

Gulden

folio

geboren

Großer Senat

Heft

Herausgeber/in

herausgegeben

Hauptstaatsarchiv Stuttgart

Jahrgang

Jahrhundert

Katholischer Kirchenrat

Kultminister/ium

Konservatorium

Evangelisches Konsistorium

Kirchenregisteramt der evangelischen Kirchenpflege

Tübingen

Kleiner Senat

Landeskirchliches Archiv Stuttgart

Landesmusikarchiv Tübingen

Ministerialabteilung

maschinenschriftlich

Die Musik in Geschichte und Gegenwart

Neue Deutsche Biographie 


$\begin{array}{ll}\text { NMZ } & \text { Neue Musikalische Zeitung } \\ \text { Nr. } & \text { Nummer } \\ \text { o. } & \text { ohne, ordentlicher } \\ \text { Phil. Fak. } & \text { Philosophische Fakultät } \\ \text { Prof. } & \text { Professor } \\ \text { RdErl. } & \text { Randerlaß } \\ \text { REM } & \text { Reichsminister(ium) für Wissenschaft, Erziehung } \\ & \text { und Volksbildung } \\ \text { S. } & \text { Seite, siehe } \\ \text { Schrb. } & \text { Schreiben } \\ \text { Sp. } & \text { Spalte } \\ \text { SR } & \text { Studienrat } \\ \text { SS } & \text { Sommersemester } \\ \text { StadtAT } & \text { Stadtarchiv Tübingen } \\ \text { StAL } & \text { Staatsarchiv Ludwigsburg } \\ \text { StBB-PK MMA } & \text { Staatsbibliothek zu Berlin, Preußischer Kulturbesitz, } \\ & \text { Musikabteilung mit Mendelssohn-Archiv } \\ \text { TC } & \text { Tübinger Chronik } \\ \text { Tl. } & \text { Teil } \\ \text { u. } & \text { und, unter } \\ \text { u.a. } & \text { und andere } \\ \text { UAT } & \text { Universitätsarchiv Tübingen } \\ \text { UBT } & \text { Universitätsbibliothek Tübingen } \\ \text { UMD } & \text { Universitätsmusikdirektor } \\ \text { Unterfasz. } & \text { Unterfaszikel } \\ \text { vgl. } & \text { vergleiche } \\ \text { WLBSt } & \text { Württembergische Landesbibliothek Stuttgart } \\ \text { WS } & \text { Wintersemester } \\ \text { x } & \text { Kreuzer } \\ \text { z.B. } & \text { zum Beispiel } \\ & \end{array}$

Zitierweise der Anmerkungen und allgemein

Die Abkürzung "aaO" steht, wenn auf die zuvor genannte Quelle verwiesen wird. Die Abkürzung "ebd." steht, wenn auf die in der vorausgehenden Anmerkung genannte Quelle und die gleiche Seite bzw. dasselbe Schriftstück verwiesen wird.

Die in den zitierten Quellen verwendete Schreibweise wurde beibehalten. Offensichtliche Verschreibungen wurden stillschweigend verbessert. Abkürzungen wurden, wenn zum Verständnis nötig, aufgelöst und in spitze Klammern gesetzt. Der Reduplikationsstrich über $m$ und $n$ führte zur Verdoppelung der Konsonanten. In eckigen Klammern stehen Ergänzungen der Verfasserin. 


\section{Ämter, Behörden und Institutionen}

\section{DEPARTEMENT}

Die unter Friedrich I. verwendete Bezeichnung für ein >MINISTERIUM. Ihm steht ein Chef (>MINISTER) vor, dem zur Verwaltung seiner Aufgaben in der Regel ein General-Sekretär und ein Kanzellist beigeordnet sind.

\section{EVANGELISCHES KONSISTORIUM}

\section{$>$ EVANGELISCHES OBERKONSISTORIUM}

\section{EVANGELISCHES OBERKONSISTORIUM}

Unter diesem Namen unterstellte Friedrich I. 1806 das in eine staatliche Aufsichtsbehörde umgewandelte Konsistorium dem > GEISTLICHEN DEPARTEMENT als Mittelbehörde. Es hatte zwar weiterhin die Aufsicht über das evangelische Kirchenund Schulwesen; tatsächlich blieb aber nur die Aufsicht über die Lateinschulen, da mit der Errichtung der > OBER-STUDIEN-DIREKTION viele Pflichten an diese neue Behörde übertragen worden waren. 1817 erfolgte die Umbenennung in EVANGELISCHES KONSISTORIUM. 1909 wurde die Schulaufsicht an den Evangelischen Oberschulrat übertragen.

\section{GEHEIMER RAT}

König Friedrich löste Ende 1805 mit der Annahme der Königswürde den altwürttembergischen Geheimen Rat auf und baute mit dem Ministerialsystem nach französischem Vorbild die Verwaltung neu auf. An die Stelle des Geheimen Rats trat das >STAATSMINISTERIUM als beratende Behörde. Wilhelm I. führte bei seinem Regierungsantritt 1816 den Geheimen Rat wieder ein, allerdings im Sinne des Staatsministeriums als oberste beratende Staatsbehörde; zusammengesetzt aus den Ministern und weiteren, vom König ernannten Mitgliedern (Staatsrat oder Geheimrat). Neben der Behandlung von Kompetenzstreitigkeiten zwischen Justiz und Verwaltungsbehörde, zwischen Kirche und Staat, diskutierte er alle Gesetzes und Verordnungsentwürfe der $>$ MINISTER und leitete sie mit seinem Gutachten an den König weiter. Bis 1848 stand dem Geheimen Rat ein hauptamtlicher Präsident vor, danach erfüllte diese Aufgabe ein Minister im Nebenamt. Als mit der Gründung des Verwaltungsgerichtshofs 1877 und des Kompetenzgerichtshofs 1879 viele Aufgaben entfielen, verlor der Geheime Rat immer mehr an Bedeutung. Endgültig aufgelöst wurde er aber erst 1911.

\section{GEISTLICHES DEPARTEMENT $>$ KULTMINISTERIUM}

\section{KABINETT}

Nach französischem Vorbild war das Königliche oder Geheime Kabinett eine Vereinigung, zu der die Minister, der Geheimratspräsident und der Staatssekretär zählten. Nach dem Ermessen und unter Vorsitz des Königs trat das Gremium in Ministerkonferenzen zur gemeinsamen Beratung zusammen. Das Kabinett hatte keine rechtliche Bedeutung, es konnte keine Beschlüsse fassen.

\section{KANZLER}

Kanzler war seit der Universitätsgründung bis 1819 jeweils der erste Professor der Theologie. Er hatte Sitz und Stimme im > SENAT und war der Vertreter des Landesherrn vor Ort. Er hatte über den Zustand der Universität und die Einhaltung der 
Satzungen zu wachen. Während des > KURATORIUMS war er zur reinen Dekoration degradiert. Mit der neuen Verfassung des Königreichs Württemberg von 1819 erhielt der Kanzler Sitz und Stimme in der zweiten Kammer der Abgeordneten. Außerdem war das Amt nicht mehr an die erste theologische Professur gebunden. Als ständiger Regierungskommissar an der Universität waren hintereinander tätig: 1819(-21 Vizekanzler)-1835 Johann Heinrich Ferdinand Autenrieth (Mediziner), 1835-1851 Karl Georg Wächter (Jurist), 1851(-55 Vizekanzler)-1862 Karl Friedrich von Gerber (Jurist), 1864-1870 Theodor von Geßler, 1870-1889 Gustav von Rümelin (Staatswissenschaftler), 1889-1899 Carl von Weizsäcker (ev. Theologe), 1899-1908 Gustav von Schönberg (Staatswirtschaftler), 1908-1931 Max von Rümelin (Jurist), 1931-1933 August Hegler (Jurist). Mit der Übernahme des Führerprinzips wurde das Amt des Kanzlers 1933 abgeschafft.

\section{KATHOLISCHER KIRCHENRAT}

Zur Bewahrung der Staatsgewalt über die katholische Kirche übertrug König Friedrich 1806 »die Bewahrung und Wahrnehmung der Souveränitätsrechte* einer Staatsbehörde, dem GEISTLICHEN RAT, von Wilhelm I. 1816 in KATHOLISCHER KIRCHENRAT umbenannt. Mit zwei weltlichen und einem geistlichen Rat, ab 1812 noch einem Direktor besetzt, war er Oberschulbehörde über die katholischen Volksschulen und Gymnasien und Aufsichtsbehörde über das Wilhelmsstift in Tübingen und als Mittelbehörde dem > KULTMINISTERIUM unterstellt. Nach der Übergabe der Leitung und Verwaltung des Wilhelmsstifts an den Bischof in Rottenburg 1934 wurde der Katholische Kirchenrat aufgelöst.

\section{KULTMINISTERIUM}

1806 von König Friedrich eingerichtetes Ministerium (mit der Bezeichnung Geistliches Departement), zu dessen Aufgabenbereich die Angelegenheiten der Kirchen und der Schulen jeder Konfession sowie des >KURATORIUMS der Universität in Tübingen (1806-1817) zählten. Die ihm untergeordneten Mittelbehörden waren das $>$ EVANGELISCHE OBERKONSISTORIUM, der > KATHOLISCHE KIRCHENRAT sowie die > OBER-STUDIEN-DIREKTION. Unter Wilhelm I. wurde das Departement 1817 umbenannt in MINISTERIUM DES KIRCHEN- UND SCHULWESENS und dem Geschäftskreis des Ministeriums des Innern beigeordnet. Erst 1848 wurden diese zwei Geschäftsbereiche wieder in zwei selbständige Ministerien getrennt. 1918, nach der Trennung von Kirche und Staat, wurde offiziell die bisherige Bezeichnung durch die schon lange gebräuchliche Bezeichnung KULTMINISTERIUM ersetzt.

\section{KURATORIUM}

Im Zuge seiner Reformen zur inneren Staatsorganisation nach französischem Vorbild verwandelte König Friedrich I. 1806 die Universität, die sich seit ihrer Gründung 1477 als autonome Körperschaft selbst verwaltet hatte, in eine Staatsanstalt. Er nahm der Hochschule Privilegien und Vermögen und unterstellte sie der Aufsicht eines KURATORS, der wiederum dem > KULTMINISTERIUM direkt untergeordnet war. 1811, als die Universität Tübingen für eine Befreiung ihrer Studenten vom Wehrdienst eintrat, hob der König kurzerhand die alte Universitätsverfassung auf. Der Kurator wurde dazu verpflichtet, Amts- und Wohnsitz in Tübingen zu nehmen. Mit der Übernahme aller Amtsgeschäfte durch den Kurator wurde sowohl die Funktion des $>$ KANZLERS als auch das Amt des > REKTORS praktisch bedeutungslos. Der Kurator übernahm sogar das bisherige Recht des Senats, Besetzungsvorschläge zu ma- 
chen. 1817 hob König Wilhelm I. die Stelle des Kurators wieder auf. Die Universität erhielt einige Rechte zurück (z.B. der Senat das Vorschlags- und Besetzungsrecht), blieb jedoch dem Kultministerium als ihrer Aufsichtsbehörde unterstellt.

\section{MINISTER}

Im engeren Sinne der ein Ministerium leitende Beamte (unter Friedrich I. Departement-Chef genannt). Er wurde vom König selbständig ernannt und war von ihm zu entlassen. In seinem Geschäftskreis war der Minister unabhängig und alleinverantwortlich tätig. Alle Angelegenheiten, die einer königlichen Entscheidung bedurften, mußte er mit schriftlichem Anbringen dem König vortragen. Dazu zählten auch Stellenbesetzungen. Der Minister hatte Sitz und Stimme im >STAATSMINISTERIUM beziehungsweise im > GEHEIMEN RAT.

\section{MINISTERIALABTEILUNG FÜR DIE HÖHEREN SCHULEN $>$ STUDIENRAT}

\section{MINISTERIALABTEILUNG FÜR GELEHRTE- UND REALSCHULEN $>$ STUDIENRAT}

\section{MINISTERIUM FÜR KIRCHEN- UND SCHULWESEN $>$ KULTMINISTERIUM}

\section{OBER-STUDIEN-DIREKTION $>$ STUDIENRAT}

\section{REKTOR}

Der > SENAT wählte aus seiner Mitte je für ein halbes Jahr den REKTOR als sein Oberhaupt. Unter seinem Vorsitz konnte sich der Senat versammeln. Während des > KURATORIUMS (1806-1817) wurde der Rektor vom Kultminister als Oberkurator ernannt und hatte dem Senat und den Fakultäten die Befehle mitzuteilen. Nach dem Ende des Kuratoriums gab es keinen Rektor mehr, bis der damalige $>$ KANZLER, Johann Heinrich Ferdinand Autenrieth, 1831 die Wiedereinführung des gewählten Rektors erwirkte; nun jeweils für ein ganzes Jahr im Amt. Der Senat schlug drei Professoren vor, aus denen der König einen ernannte. Seit 1921 wurde der Rektor vom Großen Senat gewählt und vom Staatspräsidenten nur bestätigt. Während des Dritten Reichs wurde der Rektor zum *Führer« der Universität, der die alleinige Entscheidungskompetenz besaß.

\section{SENAT}

Als oberster Behörde der Universität gehörten im 19. Jahrhundert dem Senat die Gesamtheit der ordentlichen Professoren an. Sie berieten über alle die Universität betreffenden Gegenstände. Außer der Haushaltsplanung gehörte mit zur wichtigsten Aufgabe die Besetzung der Lehrstühle, zu der der Senat das Vorschlagsrecht besaß. Die Rechte des Gremiums wurden mehrmals erheblich eingeschränkt, nicht nur während des > KURATORIUMS (1806-1817), späterhin aber auch erweitert (Vorschlagsrecht auch für die außerordentlichen Professoren, nicht nur Beratung und Vorschlagsrecht für den Haushalt, sondern auch das Recht zur Beschlußfassung). 1912 erhielt die Universität eine neue Verfassung, der zufolge der Senat in GROSSER und KLEINER SENAT (mit unterschiedlicher Besetzung, in beiden jedoch der Rektor und der Kanzler) differenziert wurde, wobei dem Großen Senat die Funktion des bisherigen Senats, 
also auch die Beratung zur Lehrstuhlbesetzung, zukam und dem Kleinen Senat mehr Verwaltungsaufgaben zufielen. Zudem gehörten nun auch alle außerordentlichen Professoren in den Großen Senat, ab 1920 auch alle Privatdozenten. Während des Dritten Reichs, von 1935 bis 1945 war der Senat faktisch außer Kraft gesetzt. Im Mai 1945 nahm er seine Arbeit wieder auf.

\section{STAATSMINISTERIUM}

Friedrich I. führte 1806 das Ministerialsystem zur Verwaltung des neuen Württembergs, der alten und der neuerworbenen Gebiete ein. An der Spitze stand das königliche Staatsministerium. Es bestand aus den Chefs der sechs > DEPARTEMENTS sowie weiteren, besonders ernannten Mitgliedern. In diesem Gremium wurden nur Dinge von allgemeinem Staatsinteresse vorgetragen, die anschließend dem König berichtet wurden. Dem Staatsministerium untergeordnet waren das Departement der Auswärtigen Angelegenheiten, Departement des Innern, Justiz-Departement, KriegsDepartement, Finanz-Departement und Geistliches Departement.

\section{STUDIENRAT}

Unter dem Namen STUDIEN-OBER-DIREKTION von Friedrich I. 1806 eingerichtete, seit 1817 unter dem Namen STUDIENRAT arbeitende Ministerialabteilung des $>$ KULTMINISTERIUMS. Sie führte die Oberaufsicht über die höheren Schulen des Landes: das Evangelische Seminar in Tübingen, die niederen Seminare (Blaubeuren, Maulbronn, Schöntal, Urach) und das Stuttgarter Gymnasium, von 1811 bis 1817 auch über die Universität Tübingen. Während dieser Zeit war der KURATOR der Universität zugleich Präsident des Studienrats. 1866 wurde der Studienrat als MINISTERIALABTEILUNG FÜR GELEHRTE- UND REALSCHULEN an das Ministerium angegliedert, ab 1903 unter der Bezeichnung MINISTERIALABTEILUNG FÜR DIE HOOHHEREN SCHULEN.

\section{SYNODUS}

Die ab 1817 SYNODE genannte Vereinigung war die Vertretung der evangelischen Kirche gegenüber dem landesherrlichen Kirchenregiment. In Verknüpfung mit ihm bestand der Synodus aus den Mitgliedern des > KONSISTORIUMS und den sechs General-Superintendenten (höheres kirchl. Aufsichtsamt, ab 1823 in Tübingen, Reutlingen, Ludwigsburg, Ulm, Heilbronn und Schwäbisch Hall). Der Synodus trat alljährlich im Herbst in Stuttgart zu seinen Beratungen zusammen. Mit der Trennung von Staat und Kirche nach dem Ersten Weltkrieg wurde die Synode zum Repräsentanten des Kirchenvolkes umgewandelt. 